\title{
Calidad de vida en usuarias del programa de tratamiento de la depresión en atención primaria
}

JULIA GONZÁLEZ(1), GLADYS YENTZEN(') y RUBÉN ALVARADO(1)

\section{RESUMEN}

El objetivo de este estudio es describir la calidad de vida de mujeres con depresión y su relación con variables sociodemográficas y propias del cuadro depresivo. Es un estudio transversal en usuarias del "Programa para la detección, diagnóstico y tratamiento integral de la depresión en Atención Primaria”, implementado en los consultorios generales urbamos del país. Se obtuvo una muestra de 201 pacientes ingresadas al Programa entre los últimos meses del año 2003 y los primeros meses del 2004, en 2 consultorios de la V Región y 5 de la Región Metropolitana. Mediante entrevistas al momento del ingreso, se aplicó una batería de instrumentos que recoge información acerca de variables sociodemográficas, sintomas depresivos (Criterios de la CIE10) y calidad de vida (Cuestionario SF-36). El puntaje promedio global de calidad de vida corresponde a 44,2 (óptimo 100). Las dimensiones con menor promedio corresponden a limitación del rol debido a problemas emocionales (27,3), salud mental (35,7), vitalidad (35,8), dolor corporal $(36,9)$ y función social $(38,7)$. De las variables sociodemográficas estudiadas, las que muestran asociación con dimensiones de la calidad de vida son escolaridad, condición laboral y participación; no así edad ni estado civil. Se observa una peor calidad de vida a mayor intensidad del cuadro depresivo. Como conclusión se determina que existe asociación entre calidad de vida y depresión, la que se refuerza al encontrar una gradiente de peor calidad de vida a mayor intensidad del cuadro, y al comparar los puntajes con población general de otros estudios. Las dimensiones más afectadas son las esperables por las características propias del cuadro depresivo.

Palabras clave: calidad de vida, depresión.

\section{ABSTRACT}

QUALITY OF LIFE IN USERS OF THE DEPRESSION TREATMENT PROGRAM IN PRIMARY ATTENTION

The purpose of this study is that of describing the quality of life of women suffering from depression and its relation to sociodemographic variables and those peculiar to depressive symptoms. It is a transversal study in users of the "Program for the detection, diagnosis and integral treatment of depression in Primary Health Care", established in the Urban General Clinics of the country. A sample of 201 patients entered the program during the closing months of 2003 and the beginning of 2004, in two outpatient departments of the $5^{\text {th }}$ Region and 5 of the Metropolitan Region. By means of interviews at the time of entry, a set of tests was applied to collect information about sociodemographic variables, depressive symptoms (Criteria of the

(1) Escuela de Salud Pública. Facultad de Medicina. Universidad de Chile. juliagonzalez@med.uchile.cl 
CIE-10) and quality of life (Questionnaire SF-36). The average global grading for quality of life corresponds to 44.2 (optimal 100). Lower average dimensions correspond to role limitations due to emotional problems (27.3), mental health (35.7), vitality (35.8), bodily pains (36.9) and social role (38.7). Of the sociodemographic variables studied, those associated to quality of life dimensions are schooling, working condition and participation; age and civil status are not included. The higher the intensity of depressive manifestations, the lower is the quality of life observed. As a conclusion, it has been determined that there exists a link between quality of life and depression, which is reinforced when observing a worse quality of life gradient, the greater the intensity of depressive manifestations, and when comparing the grading with the general population of other studies. The more affected dimensions are those to be expected due to the inherent characteristics of depressive manifestations.

Key words: quality of life, depression.

\section{INTRODUCCIÓN}

La calidad de vida se ha constituido progresivamente en una preocupación tanto a nivel individual como poblacional en múltiples sectores. Se la define como la percepción de un individuo de su posición en la vida, en el contexto cultural y el sistema de valores en que vive, en relación con sus metas, objetivos, expectativas, valores y preocupaciones (OMS, grupo WHOQOL, 1994). En una concepción acotada al campo de la salud, tanto a nivel clínico como colectivo, se hace referencia a la "calidad de vida relacionada con la salud", la que se concibe como la percepción subjetiva de los efectos de una enfermedad o un tratamiento sobre diferentes ámbitos de la vida. Durante las últimas décadas se han acumulado, a nivel internacional, propuestas metodológicas para su medición y se ha tomado conciencia de los usos potenciales de esta variable t-3. $^{1-3}$.

Una muestra del interés creciente en nuestro país respecto de la calidad de vida relacionada con la salud, ha sido la realización, durante el año 2000, de la I Encuesta de Calidad de Vida y Salud por parte del Ministerio de Salud de Chilet.

Se reconoce a la calidad de vida, en su concepción general, como uno de los tantos determinantes del nivel de salud de la población; y, por otra parte, la existencia de una patología crónica influirá sobre la calidad de vida relacionada con la salud; en este caso, la calidad de vida aparece como determinada por los procesos de salud enfermedad.

Por otra parte, la depresión constituye en Chile un problema de salud de alta prevalencia, con consecuencias en la salud física, en la vida familiar y en el costo de la atención. En población general, sobre los 15 años, se estima una prevalencia de vida de depresión mayor alrededor del $9 \%$ y en consultantes de atención primaria aproximadamente un tercio sufre cuadros depresivos clínicos o subclínicos. En relación a la distribución por edad, se observa mayor prevalencia entre 20 y 45 años; en mujeres la frecuencia es el doble que en hombres, ocupando la depresión el segundo lugar en el estudio de carga de enfermedad en mujeres ${ }^{5,6}$.

El Ministerio de Salud de Chile ha implementado desde el año 2001, el "Programa para la detección, diagnóstico y tratamiento integral de la depresión en Atención Primaria", que considera detección, diagnóstico, registro, tratamiento médico, farmacológico y psicosocial y seguimiento de cada caso ${ }^{7}$. Desde el año 2003, el Programa se extendió a todo el país, por lo que se consideró importante realizar una evaluación de éste, que involucrara el seguimiento de un grupo de pacientes durante el primer semestre de tratamiento. Cabe señalar que la gran mayoría de los usuarios de este programa son mujeres $(92 \%)$

Uno de los objetivos de este Programa es mejorar la calidad de vida y el bienesta subjetivo de las ustuarias. Por este motivo, nos interesó describir la calidad de vida de las 
mujeres al momento de ingresar a él, y su relación con variables sociodemográficas y clínicas propias del cuadro depresivo, con el fin de contribuir a un mejor conocimiento de las relaciones mutuas entre depresión y calidad de vida.

\section{MATERIAL Y MÉTODO}

Se trata de un estudio transversal descriptivo, relativo a la calidad de vida de las mujeres, al momento del ingreso al Programa para la Detección, Diagnóstico y Tratamiento Integral de la Depresión en Atención Primaria.

Este estudio forma parte de una evaluación más amplia de este programa y que involucra el seguimiento de un grupo de pacientes durante el primer semestre de tratamiento.

\section{Universo y muestra}

Se seleccionaron 2 consultorios de la V Región y 5 de la Región Metropolitana, en que el Programa mostraba un buen nivel de funcionamiento. En estos centros, se conformó una muestra de 201 pacientes, en la medida que iban ingresando al Programa, entre los últimos meses del año 2003 y los primeros del año 2004.

Los criterios de inclusión fueron: ser mujer; tener entre 25 y 65 años; no presentar un déficit sensorial ni cognitivo que limite la posibilidad de comunicación con el entrevistador; no estar embarazada ni haber tenido su parto en los dos meses previos a su ingreso; tener residencia estable y acceso telefónico.

\section{Descripción de la muestra}

El rango de edad fluctuó entre los 25 y 65 años, con mediana y promedio de $44,0 \pm 11$ años. En cuanto a su estado civil, se encuentra un $15 \%$ de solteras, $48 \%$ casadas, $15 \%$ convivientes, $16 \%$ separadas y $5 \%$ viudas; de modo que dos tercios de la muestra declara tener pareja estable.

En relación al nivel educacional, la relación es de $1 \%$ sin estudios, $40 \%$ con educación básica, $41 \%$ educación media, $12 \%$ educación técnica y $5 \%$ universitaria. Se encuentra, además, que la mediana de años de estudio aprobados es de 10 .
El $34,0 \%$ de las mujeres tiene un trabajo remunerado; de éstas el $52,9 \%$ tiene un trabajo estable fuera de la casa y el $22,1 \%$ un trabajo ocasional fuera de la casa.

Respecto de participación social, el 65,3\% de las mujeres no participa en ningún grupo social, $13,1 \%$ lo hace en forma ocasional $y$ $21,6 \%$ lo hace frecuentemente en, al menos, una organización. Este último grupo de mujeres concentra su participación en iglesia $(46,3 \%)$ y juntas de vecinos $(30,1 \%)$

De los factores psicosociales estudiados, los más frecuentemente identificados fueron el antecedente de un episodio depresivo anterior $(65,6 \%)$ y la coexistencia de una enfermedad física o dolor crónico $(45,5 \%)$.

El diagnóstico clínico de intensidad del cuadro depresivo efectuado por la entrevistadora, de acuerdo a la CIE-10, muestra un $11,9 \%$ de mujeres con depresión leve, $62,7 \%$ moderada y $25,4 \%$ severa. Estos resultados son coherentes con los del Inventario de Depresión de Beck (IDB).

\section{Variables, instrumentos y recolección de la información}

A todas las mujeres se les informó en qué consistía el estudio, se les solicitó firmar un consentimiento informado.

Para recolectar la información se realizaron entrevistas cara a cara al ingreso, por dos profesionales mujeres, especialmente entrenadas para este estudio.

Se utilizó una batería de instrumentos que recogen información acerca de variables sociodemográficas, síntomas depresivos y calidad de vida:

Para obtener datos sociodemográficos, tales como edad, estado civil, escolaridad, trabajo y participación social, se utilizó una encuesta diseñada especialmente para este estudio.

Para realizar el diagnóstico clínico de la intensidad del cuadro depresivo se aplicaron los criterios de la CIE - 10 para la Atención Primaria, de acuerdo a la guía de práctica clínica del Ministerio de Salud ${ }^{7}$; éstos permiten clasificar a las pacientes en depresión leve, moderada y severa. Adicio- 
nalmente, se usó el Inventario de Depresión de Beck (IDB) $)^{8,9}$.

El instrumento utilizado para medir calidad de vida corresponde al Cuestionario de Salud SF-361. Este es un instrumento genérico ampliamente usado y validado a nivel internacional.

El SF-36 fue desarrollado para su uso en el Estudio de Resultados Médicos (Medical Outcomes Study, Ware y Sherbourne, 1992) como una medida genérica de calidad de vida relacionada con la salud. El cuestionario es aplicable tanto a población general como a pacientes, en estudios descriptivos y de evaluación. Incluye escalas multi item para medir 8 dimensiones, que cubren estado funcional, bienestar emocional y salud general; en este estudio se considera tanto la puntuación global de calidad de vida, como los puntajes obtenidos para cada dimensión.

Las dimensiones relativas a estado funcional son funcionamiento físico, funcionamiento o desempeño social, limitación del rol debido a problemas de salud física, limitación del rol debido a problemas emocionales. Las dimensiones relativas a bienestar son salud mental, que incluye distrés psicológico y bienestar; vitalidad, energía o fatiga; y dolor corporal. Por último, se agrega una dimensión relativa a salud general: percepción general de su salud.

Cada una de las 8 dimensiones está representada en el cuestionario por un número variable de preguntas (entre 2 y 10); y cada una de las preguntas cuenta con entre 3 y 6 alternativas.
Para el análisis de los resultados del SF-36 se usa el enfoque Rand": mediante éste, se lleva el puntaje de cada dimensión a escala de 0 a 100; a mayor puntuación, mejor calidad de vida relacionada con la salud. A pesar de que el cuestionario no fue diseñado para calcular un puntaje global, algunos investigadores han calculado promedios de puntaje para las áreas de salud física y de salud mental ${ }^{1}$; en este estudio se optó por calcular un puntaje global que corresponde al promedio de los puntajes de las 8 dimensiones, teniendo igualmente un máximo de 100 (lo mejor) y un mínimo de 0 (lo peor).

Se estudió la asociación entre los puntajes del SF-36 globales y de sus 8 dimensiones, con las variables sociodemográficas y con la intensidad de la depresión. Con el fin de analizar la asociación entre calidad de vida y participación, la participación se clasificó como "sí" cuando la persona participa al menos en una organización y en forma frecuente; y como "no" cuando no participa en ninguna o sólo lo hace ocasionalmente.

Para comparar los promedios en el SF-36 respecto a categorías de variables cualitativas se usó comparación de promedios a través de la prueba T o análisis de varianza; cuando no se cumplía el supuesto de homogeneidad de la varianza (Prueba de Bartlett) se utilizó la prueba de Kruskall Wallis.

Para comparar el puntaje del SF-36 respecto de variables cuantitativas se usó la correlación de Pearson. El nivel de significación considerado fue de 0,05 .

Tabla 1. Calidad de vida al ingreso al programa. Puntajes del Cuestionario SF-36

\begin{tabular}{|c|c|c|c|c|}
\hline \multirow[t]{2}{*}{ Dimensiones } & \multirow[b]{2}{*}{ Mínimo } & \multicolumn{3}{|c|}{ Puntajes del SF-36 } \\
\hline & & Máximo & Mediana & Promedio $\pm \mathrm{DS}$ \\
\hline Función física $(\mathrm{FF})$ & 10,0 & 95,0 & 55,0 & $51,6 \pm 27,6$ \\
\hline Limit.rol salud física (LRSF) & 0,0 & 100,0 & 50,0 & $49,2 \pm 44,9$ \\
\hline Dolor corporal (DC) & 0,0 & 90,0 & 35,0 & $36,9 \pm 23,3$ \\
\hline Función social (FS) & 0,0 & 88,0 & 38,0 & $38,7 \pm 25,8$ \\
\hline Salud mental (SM) & 0,0 & 88,0 & 36,0 & $35,7 \pm 17,2$ \\
\hline Limit.rol problenoc. (LRPE) & 0,0 & 100,0 & 0,0 & $27,3 \pm 40,5$ \\
\hline Vitalidad y energía (VE) & 0,0 & 90,0 & 35,0 & $35,8 \pm 18,4$ \\
\hline Percep.global salud (PGS) & 0,0 & 90,0 & 40,0 & $40,9 \pm 17,7$ \\
\hline Calidad de vida global (CVG) & 5,4 & 93,1 & 43,6 & $44.2 \pm 18,5$ \\
\hline
\end{tabular}


Tabla 2. Calidad de vida según escolaridad. Puntajes del Cuestionario SF-36

\begin{tabular}{|c|c|c|c|}
\hline \multirow[t]{2}{*}{ Dimensiones } & \multicolumn{2}{|c|}{ Escolaridad en años de estudio } & \multirow[t]{2}{*}{$\mathbf{P}$} \\
\hline & 0 a 8 & 9 y más & \\
\hline Función física (FF) & $50,7 \pm 26,8$ & $52,2 \pm 28,2$ & 0,7052 \\
\hline Limit.rol salud fisica (LRSF) & $46,0 \pm 44,1$ & $51,4 \pm 45,5$ & 0,4259 \\
\hline Dolor corporal (DC) & $40,1 \pm 25,3$ & $34,7 \pm 21,7$ & 0,1224 \\
\hline Función social (FS) & $42,2 \pm 25,3$ & $36,3 \pm 25,7$ & 0,1284 \\
\hline Salud mental (SM) & $37,9 \pm 16,9$ & $34,2 \pm 17,4$ & 0,1526 \\
\hline Limit.rol probl.emoc. (LRPE) & $36,0 \pm 44,5$ & $21,2 \pm 36,5$ & 0,0247 \\
\hline Vitalidad y energía (VE) & $39,9 \pm 16,9$ & $32,9 \pm 17,9$ & 0,0113 \\
\hline Percep.global salud (PGS) & $39,9 \pm 16,9$ & $41,5 \pm 18,3$ & 0,5482 \\
\hline Calidad de vida global (CVG) & $46,2 \pm 19,7$ & $42,7 \pm 17,6$ & 0,2178 \\
\hline
\end{tabular}

Tabla 3. Calidad de vida según condición laboral. Puntajes del Cuestionario SF-36

\begin{tabular}{|c|c|c|c|}
\hline \multirow[t]{2}{*}{ Dimensiones } & \multicolumn{2}{|c|}{ Condición laboral } & \multirow[t]{2}{*}{$\boldsymbol{P}$} \\
\hline & No trabaja & Trabaja & \\
\hline Función física $(\mathrm{FF})$ & $51,6 \pm 27,1$ & $51,9 \pm 28,6$ & 0,9311 \\
\hline Limit.rol salud física (LRSF) & $49,6 \pm 45,0$ & $49,2 \pm 45,1$ & 0,9614 \\
\hline Dolor corporal (DC) & $36,3 \pm 21,9$ & $38,5 \pm 25,5$ & 0,5400 \\
\hline Función social (FS) & $35,8 \pm 26,6$ & $43,8 \pm 23,8$ & 0,0449 \\
\hline Salud mental (SM) & $34,4 \pm 17,5$ & $38,2 \pm 16,7$ & 0,1627 \\
\hline Limit.rol probl.emoc. (LRPE) & $25,0 \pm 38,8$ & $31,8 \pm 43,5$ & 0,2793 \\
\hline Vitalidad y energia (VE) & $36,1 \pm 19,1$ & $35,5 \pm 17,0$ & 0,8502 \\
\hline Percep.global salud (PGS) & $39,2 \pm 17,3$ & $44,0 \pm 18,4$ & 0,0825 \\
\hline Calidad de vida global (CVG) & $43,0 \pm 18,9$ & $46,6 \pm 17,6$ & 0,2102 \\
\hline
\end{tabular}

Tabla 4. Calidad de vida según participación. Puntajes del Cuestionario SF-36

\begin{tabular}{|c|c|c|c|}
\hline \multirow[t]{2}{*}{ Dimensiones } & \multicolumn{2}{|c|}{ Participación social } & \multirow[t]{2}{*}{$\mathbf{P}$} \\
\hline & No participa & Participa & \\
\hline Función física (FF) & $51,4 \pm 27,0$ & $54,6 \pm 29,4$ & 0.4948 \\
\hline Limit rol salud física (LRSF) & $48.6 \pm 45.7$ & $51,3 \pm 43,5$ & 0,7377 \\
\hline Dolor corporal (DC) & $35,9 \pm 23,3$ & $41,7 \pm 22,0$ & 0,1481 \\
\hline Función social (FS) & $38,3 \pm 24,9$ & $43,1 \pm 27,6$ & 0.2721 \\
\hline Salud mental (SM) & $33.5 \pm 15.8$ & $43,9 \pm 18,8$ & 0,0004 \\
\hline Limit.rol probl.emoc. (L.RPE) & $25,3 \pm 39,3$ & $39,7 \pm 46,1$ & 0,0435 \\
\hline Vitalidad y energia ( VE) & $35,4 \pm 18,4$ & $41,2 \pm 18,8$ & 0,0699 \\
\hline Percep.global salud (PGS) & $40,2 \pm 16.7$ & $43,7 \pm 19,7$ & 0,2415 \\
\hline Calidad de vida global (CVG) & $42,6 \pm 17,7$ & $50,2 \pm 20,6$ & 0,0267 \\
\hline
\end{tabular}

\section{RESULTADOS}

\section{Calidad de vida medida mediante el Cuestionario SF-36}

En la Tabla 1 se muestran los puntajes del SF-36 para las diferentes dimensiones de calidad de vida. El puntaje promedio global de calidad de vida corresponde a 44,2 , con un rango entre 5,4 y 93,1. Las dimensiones con puntaje promedio más bajo corresponden a limitación del rol debido a problemas emocionales (27,3), salud mental $(35,7)$, vitalidad $(35,8)$, dolor corporal $(36,9)$ y función social $(38,7)$. Llama la atención que para 
Tabla 5. Calidad de vida según intensidad del cuadro depresivo. Puntajes del Cuestionario SF-36

\begin{tabular}{lccrr}
\hline Dimensiones & Leve & $\begin{array}{c}\text { Intensidad del cuadro depresivo } \\
\text { Moderada }\end{array}$ & Severa & P \\
\hline Función física & $52,8 \pm 30,7$ & $50,2 \pm 28,0$ & $54,0 \pm 25,3$ & 0,7089 \\
Limit.rol salud física & $73,9 \pm 36,5$ & $52,8 \pm 44,5$ & $30,0 \pm 42,3$ & 0,0002 \\
Dolor corporal & $50,1 \pm 20,2$ & $37,9 \pm 23,2$ & $28,7 \pm 22,1$ & 0,0008 \\
Función social & $46,0 \pm 40,3$ & $40,3 \pm 25,3$ & $31,9 \pm 24,3$ & 0,0559 \\
Salud mental & $52,3 \pm 14,4$ & $36,7 \pm 16,1$ & $26,1 \pm 14,3$ & 0,0001 \\
Limit.rol probl.emoc. & $56,5 \pm 46,6$ & $25,4 \pm 38,5$ & $18,0 \pm 36,4$ & 0,0005 \\
Vitalidad y energía & $46,3 \pm 21,2$ & $36,6 \pm 18,0$ & $29,2 \pm 15,3$ & 0,0007 \\
Percep.global salud & $57,2 \pm 15,3$ & $42,2 \pm 16,1$ & $30,5 \pm 15,6$ & $<0,0001$ \\
Calidad de vida global & $62,7 \pm 15,2$ & $44,9 \pm 16.3$ & $33,9 \pm 17,6$ & $<0,0001$ \\
\hline
\end{tabular}

Tabla 6. Calidad de vida en población en estudio y población general de diferentes países. Puntajes promedio y D.S. del Cuestionario SF-36

\begin{tabular}{lcccr}
\hline Dimensiones & U.S.A. (2)* & España (12)** & México (11)*** & EstudioActual \\
\hline Función física & $84,2 \pm 23,3$ & $81,5 \pm 25,7$ & $94,0 \pm 9,3$ & $51,6 \pm 27,6$ \\
Limit.rol salud física & $81,0 \pm 34,0$ & $79,5 \pm 38,0$ & $95,0 \pm 15,1$ & $49,2 \pm 44,9$ \\
Dolor corporal & $75,2 \pm 23,7$ & $74,4 \pm 29,7$ & $90,0 \pm 16,3$ & $36,9 \pm 23,3$ \\
Función social & $83,3 \pm 22,7$ & $87,9 \pm 21,7$ & $87,0 \pm 15,7$ & $38,7 \pm 25,8$ \\
Salud mental & $74,7 \pm 18,1$ & $70,1 \pm 20,9$ & $80,0 \pm 14,4$ & $35,7 \pm 17,2$ \\
Limit. Rol problema & $81,3 \pm 33,0$ & $84,4 \pm 34,2$ & $87,0 \pm 20,4$ & $27,3 \pm 40,5$ \\
Emocional & & & & \\
Vitalidad y energía & $60,9 \pm 21,0$ & $63,6 \pm 22,6$ & $74,0 \pm 14,8$ & $35,8 \pm 18,4$ \\
Percep.global salud & $72,0 \pm 20,3$ & $65,9 \pm 22,8$ & & $40,9 \pm 17,7$ \\
\hline
\end{tabular}

* U.S.A. : población general de ambos sexos.

** España: población general de sexo femenino.

*** México: mujeres de 18 a 64 años, trabajadoras de institución pública, que no han utilizado servicios médicos en el último año y no padecen enfermedad crónica.

limitación del rol debido a problemas emocionales la mediana es cero, indicando una gran limitación en esta dimensión.

\section{Relación entre calidad de vida y variables sociodemográficas}

No se observa asociación entre calidad de vida global, o alguna de sus dimensiones, con la edad ni con el estado civil de las pacientes.

En la Tabla 2 se muestra la relación entre calidad de vida y escolaridad. Si bien no se observa relación entre calidad de vida global y escolaridad, entre las mujeres con más de 9 años de estudios se encuentra un puntaje significativamente menor para las dimensiones limitaciones del rol debido a problemas emocionales, así como para vitalidad y energía.

En la Tabla 3 se presenta la relación entre calidad de vida y condición laboral. No se observa relación entre calidad de vida global y trabajo; sin embargo, en mujeres que no trabajan, se encuentra un puntaje significativamente menor para la dimensión funcionamiento social.

La relación entre calidad de vida y participación, se presenta en la Tabla 4 . El puntaje es significativamente menor en las mujeres que no participan o que lo hacen en forma ocasional, tanto para calidad de vida global, como para las dimensiones salud mental y limitación del rol para problemas emocionales.

\section{Relación entre calidad de vida e intensidad del cuadro depresivo}

Al relacionar calidad de vida e intensidad 
de la depresión de acuerdo al diagnóstico de la entrevistadora, se observa que a mayor gravedad del cuadro, menor puntaje en calidad de vida; además de encontrarse una gradiente para las categorías de gravedad, las diferencias son estadísticamente significativas. Como se observa en la Tabla 5, lo anterior es válido tanto para calidad de vida global, como para cada una de sus dimensiones, excepto funcionamiento físico.

\section{DISCUSIÓN}

\section{Limitaciones del estudio}

Por tratarse de un estudio de tipo transversal, no es posible extraer conclusiones respecto de la direccionalidad de las asociaciones encontradas. En este sentido se espera a futuro analizar las variaciones de la calidad de vida en la medida que avanza el tratamiento $y$, consecuentemente, disminuye la gravedad del cuadro clínico.

La extrapolación de los resultados al total de pacientes en control en el Programa podría verse limitada por el hecho de que la selección de los consultorios no fue aleatoria. Adicionalmente, las características de la población en estudio - mujeres de edad media, con depresión, atendidas en centros de atención primaria del sistema público de salud - no permiten extrapolar los resultados a un universo más amplio.

En la relación que pudiese existir entre calidad de vida y depresión pueden identificarse otras variables intervinientes. Por ejemplo, en la literatura se ha descrito un deterioro de la calidad de vida en mujeres climatéricas ${ }^{10}$, variable que podría estar influyendo en nuestros resultados dado que la mediana de edad de la muestra fue de 44,0 años. A futuro sería de interés analizar las relaciones mutuas entre climaterio, depresión y calidad de vida.

\section{Calidad de vida}

Al analizar los resultados del SF-36 al ingreso de las pacientes, destaca que tanto la medición global como sus dimensiones se encuentran muy por debajo del óptimo, lo cual es coherente con lo esperado para un cuadro clínico que afecta múltiples aspectos de la vida de las pacientes. Las dimensiones menos afectadas tienen relación con la salud física. La baja puntuación en la dimensión de dolor corporal se puede explicar a través de los resultados de la investigación clínica y psicofisiológica que ha demostrado que las personas que sufren un cuadro depresivo tienen un menor umbral y tolerancia al dolor. La dimensión más afectada, que corresponde a limitaciones del rol por problemas emocionales, muestra un puntaje promedio de 27,3 con una mediana de 0 ; esto implica que, al menos, la mitad de las mujeres ha hecho menos, durante menos tiempo y menos cuidadosamente que lo esperado y ha visto extremadamente limitadas sus actividades sociales, debido a algún problema emocional.

Dado que aún no se dispone de parámetros nacionales de referencia para el Cuestionario SF-36, en la Tabla 6 se comparan los resultados con parámetros de otros países en que este instrumento ha sido validado: población general de U.S.A., población general femenina de España y mujeres sanas de edad media de México2,11,12.

Como se aprecia en la Tabla 6 , las usuarias al ingresar al Programa, presentan una calidad de vida mucho más baja que la población general en todas sus dimensiones; $y$, en particular, en el desempeño del rol por problemas emocionales, lo cual es coherente con el tipo de problema de salud estudiado.

Al comparar los resultados de la medición de calidad de vida con un estudio chileno en mujeres con depresión severa, al momento del ingreso a un programa similar ${ }^{13}$, se encuentra una mejor calidad de vida en el presente estudio. Las dimensiones más afectadas coinciden en ambos casos, pero los puntajes son muy inferiores en el estudio citado, para las dimensiones salud mental, limitación del rol por problemas emocionales y vitalidad. Esto podría explicarse por una diferente intensidad del cuadro depresivo en las dos muestras.

\section{Relación con variables sociodemograficas}

De las variables estudiadas, la que muestra una mayor asociación con calidad de vida corresponde a participación; el grupo de 
mujeres que participa en forma frecuente en alguna organización social presenta menor percepción de compromiso de su salud mental y de limitaciones de su rol por problemas emocionales. Sería de interés a futuro confirmar la hipótesis de que la participación social constituye un factor protector de la calidad de vida en mujeres depresivas; otra posibilidad sería que se seleccionaran las pacientes con mejor calidad de vida para participar en alguna organización.

La segunda variable en que se observa asociaciones significativas es la escolaridad; en este caso, las mujeres con menos de 9 años de estudio perciben menos limitaciones del rol por problemas emocionales y menor compromiso de su vitalidad y energía que aquellas con mayor escolaridad. Esto podría explicarse porque las mujeres con más años de estudio tendrían mayores expectativas de desempeño en diferentes ámbitos de su vida; lo cual determinaría una mayor distancia entre lo que perciben tener y sus expectativas.

\section{Relación con intensidad del cuadro depresivo}

La asociación entre la intensidad del cuadro depresivo y el deterioro en la calidad de vida se confirma al analizar los puntajes del SF-36 para los distintos subgrupos clínicos de depresión. Todas las dimensiones, salvo función física, muestran una gradiente de peor calidad de vida a mayor severidad de la depresión; las diferencias observadas son altamente significativas. Esto es concordante con las diferencias observadas entre nuestras pacientes depresivas y la población presuntamente sana.

\section{REFERENCIAS}

1.- BADIA X, SALAMERO M, ALONSO J. La Medida de la Salud; Edimac, Barcelona 2002.

2.- MCDOWELL 1, NEWELL C. Measuring Health; Oxford University Press, 1996.

3.- FORATTINI O. Qualidade de vida e meio urbao. A cidade de Sao Paulo, Brasil. Rev Saude Publ S Paulo 1991; 25(2): 75-86.

4.- AgUILERA $X$, et al. Unidad de Estudio. Departamento de Epidemiología. Salinas J. y cols. Departamento de Promoción. División de Rectoría y Regulación Sanitaria, Ministerio de Salud, Chile. Encuesta Nacional de Calidad de Vida y Salud, Chile 2000, INE - Minsal).

5.- ALVARADO R, et al. Prevalencia y detección de cuadros depresivos en pacientes adultas de centros de atención primaria, Chiloé. Rev Chil Salud Pública $2004 ; 8(3): 126-36$.

6.- VICENTE P, BENJAMÍN, et al. Estudio chileno de prevalencia de patología psiquiátrica (DSM-HI-R/ CIDI) (ECPP). Rev Méd Chile 2002; 130; n5, Santiago mayo 2002.

7.- MINISTERIO DE SALUD. Guía Clínica para la Atención Primaria en la Detección, Diagnóstico y Tratamiento de la Depresión, Chile.

8.- BECK A T, et al. An inventory on meassuring depression. Arch Gen Psychiatry 1961; 4: 53-63.

9.- BECK A T, et al. Psychometric properties of the Beck Depression Inventory. Clinical Psychology Review 1988; 8: 78.100.

10. BINFA L. Impacto del climaterio en la calidad de vida de las mujeres, Tesis para optar al grado de Magister en Salud Pública, Escuela de Salud Pública, Universidad de Chile, 1998.

11. ZÚNIIGA A, et al. Evaluación del estado de salud con la encuesta SF-36: resultados preliminares en México, Salud Pública de México 1999; 41(2), Marzo - Abril.

12.- ALONSO J, et al. Valores poblacionales de referencia de la versión española del Cuestionario de Salud SF36. Med. Clin. (Barc.) 1998; 111: 410-6.

13. - ARAYA R, ROJAS G, et al. Treating depression in primary care in low-income women in Santiago, Chile: a randomised controlled trial. The Lancet $2003 ; 361(22)$ : $995-1000$.

Usted puede comentar éste y otros artículos publicados en la Revista Chilena de Salud Pública, enviando un correo electrónico a revistasp@med.uchile.cl 